\title{
Acupuncture for the relief of odynophagia and xerostomia after chemoradiation therapy in oropharyngeal cancer: a case report
}

\author{
Berdale Colorado ${ }^{1}$ and Hong $\mathrm{Wu}^{1,2^{*}}$ \\ *Correspondence: hongwu@mcw.edu \\ 'Department of Physical Medicine \& Rehabilitation Medical College of Wisconsin, USA. \\ 2Department of Anesthesiology Medical College of Wisconsin, USA.
}

\begin{abstract}
Objectives/Case description: A 48 year old male presented with 6-month history of odynophagia and xerostomia, which began during chemotherapy (cetuximab) and radiation therapy (66 Gray in 33 fractions) for treatment of clinical stage T1N1M0 squamous cell carcinoma of his tongue. Odynophagia was described as aching pain at the base of his tongue that felt like his throat was swollen, and was worse with swallowing. He also reported xerostomia, with associated dysphagia and impaired taste. The patient had failed pilocarpine, Biotene rinse, and speech therapy. Physical examination revealed dry oral mucous membranes and skin hyperpigmentation on the neck, consistent with radiation therapy. The musculoskeletal and neurological exam of the head, neck, and upper extremities were otherwise unremarkable.

Methods: He received weekly 20-minute sessions of acupuncture (using combination of manual, auricular, and electro-acupuncture) for 10 sessions. He completed a self-report questionnaire asking about the percentage improvement of his symptoms of pain, dry mouth, difficulty swallowing, taste impairment, insomnia, and anxiety at each session.

Results: He reported resolution of odynophagia after 4 acupuncture sessions, with the most dramatic improvement after session \#2 (50\%) and session \#3 (90\%). He reported gradual improvement of xerostomia, with 30-40\% improvement, over 10 acupuncture sessions. Gradual improvement over 10 acupuncture sessions was noted in symptoms associated with xerostomia, such as ability to swallow (25-30\% improvement), and sense of taste (25-30\% improvement).
\end{abstract}

Discussion: Odynophagia and xerostomia are common complications after chemoradiation in oropharyngeal cancer, and can be difficult to manage. This case report found that acupuncture was effective in treating odynophagia and xerostomia after chemoradiation in oropharyngeal cancer.

Key words: Acupuncture, deglutition disorders, oropharyngeal neoplasms, xerostomia

\section{Introduction}

Head and neck cancer accounts for approximately 3 percent of all cancers in the United States. Over 52,000 people in the United States are expected to be diagnosed with head and neck cancers in 2012 [1]. Treatment of head and neck cancer commonly involves radiotherapy, with or without concomitant chemotherapy [2]. Radiation therapy damages the mucosa and soft tissue within the radiation treatment volume, resulting in an inflammatory reaction and the production of reactive oxygen species [3]. Odynophagia (painful swallowing) and xerostomia (lack of saliva) are common side effects following chemoradiation therapy in head and neck cancer. Xerostomia is the most frequent complication reported after head and neck radiotherapy; however, odynophagia is considered as the most detrimental component of quality of life [4]. Tissue swelling, odynophagia, and xerostomia all contribute to dysphagia. Odynophagia secondary to radiation-induced mucositis typically resolves within 3 weeks of cessation of chemoradiation in most patients [5].
Current standard treatment options for odynophagia include topical and systemic analgesics (opioid and nonopioid). There is limited data on the effectiveness of these agents for pain relief in patients with head and neck cancer following chemoradiation therapy. The use of opioid analgesics can produce common adverse effects such as constipation, sedation, nausea and vomiting. Current standard treatment options for xerostomia include saliva substitutes and stimulating agents, such as pilocarpine hydrochloride (a cholinergic agonist). However, saliva substitutes are generally ineffective and drugs other than pilocarpine have provided no proven clinical benefit [6]. The reported response rate for pilocarpine is 31\%-54\% [6].

Acupuncture has long been used for a variety of medical problems, and is considered a relatively safe modality [7]. It has been found in previous studies to be effective in reducing dysphagia symptom severity in head and neck cancer patients [8-11]. Acupuncture has also been found in previous studies to be effective in stimulating saliva and improving xerostomia [12-16].

C 2013 Wu et al; licensee Herbert Publications Ltd. This is an Open Access article distributed under the terms of Creative Commons Attribution License (http://creativecommons.org/licenses/by/3.0). This permits unrestricted use, distribution, and reproduction in any medium, provided the original work is properly cited 


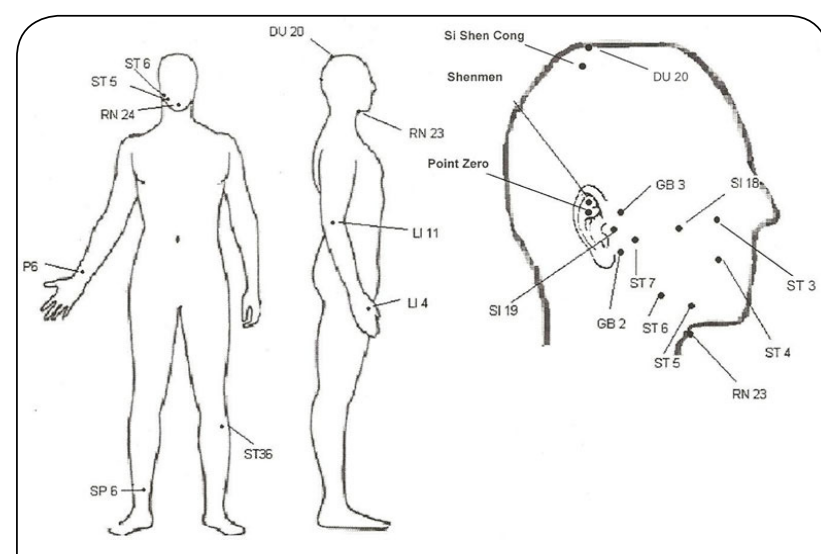

Figure 1. Acupuncture points were localized to the head, ears, arms and legs.

Although previous studies have reported the use of acupuncture on dysphagia and xerostomia following chemoradiation therapy in head and neck cancer; to our knowledge, no one has previously reported the use of acupuncture looking specifically at the impact on odynophagia, or painful swallowing. In this case study, we report resolution of odynophagia related to chemoradiation therapy for oropharyngeal cancer in a patient treated with acupuncture. Additionally, in this case study, we report significant improvement of xerostomia related to chemoradiation therapy for oropharyngeal cancer in a patient treated with acupuncture. Specifically, the patient was treated using a combination of manual acupuncture, auricular acupuncture, and electro-acupuncture. This combination has been demonstrated in a prior case study [12] as an effective treatment for xerostomia related to radiotherapy for head and neck cancer. To our knowledge, no one has previously reported using a combination of manual acupuncture, auricular acupuncture, and electroacupuncture to treat radiation-induced odynophagia.

\section{Case presentation}

The patient is a 48 year old male with clinical stage T1N1M0 squamous cell carcinoma of his tongue. He was treated with concurrent chemotherapy (with cetuximab) and radiation therapy ( 66 Gray in 33 fractions). The patient reported odynophagia and xerostomia which began during chemoradiation therapy and persisted 5 months after its completion. Odynophagia was described as aching pain at the base of his tongue and throat that felt like his throat was swollen, and was worse with swallowing. He also reported xerostomia, with associated dysphagia, and decreased sense of taste. His odynophagia and xerostomia interfered with his sleep and affected his occupation as a lawyer, due to his difficulty speaking. The patient had tried pilocarpine, Biotene ${ }^{\circledast}$ rinse (GlaxoSmithKline, United States), and speech therapy for his odynophagia and xerostomia with minimal improvement. Physical examination revealed dry oral mucous membranes and skin hyperpigmentation on the neck, consistent with radiation therapy. There were no areas of skin breakdown or irritation and no lymphedema noted. The musculoskeletal and neurological exam of the head, neck, and upper extremities were otherwise unremarkable.

\section{Methods}

He was referred to our acupuncture clinic and received a total of 10 treatments in a period of 10 weeks. He received no other co-interventions for odynophagia, such as analgesics, or for xerostomia, such as pilocarpine or saliva substitutes, during acupuncture treatments. A physician board certified in Physical Medicine and Rehabilitation as well as Eastern medicine (including acupuncture) performed all acupuncture treatments at her acupuncture clinic. Prior to acupuncture treatment, it was ascertained that patient did not have any contraindications for acupuncture treatment in a cancer patient (such as being immunocompromised; having any skin infections/disorders or bleeding disorders; or history of axillary dissection on ipsilateral side of proposed treatment) or electro-acupuncture (such as presence of cardiac pacemaker) [17]. Manual acupuncture, electro-acupuncture and auricular acupuncture were used in each session. Clean technique was utilized throughout the treatments. The acupuncture points used were in the scalp, and bilateral face, ears, arms and legs. Treatment points included ST 3, 4, 5, 6, 7, and 36, SI 18 and 19, GB 2 and 3, LI 4 and 11, SP 6, DU 20, RN 23 and 24, and the ear points, Shenmen and point zero (Figure 1). The acupuncture points used in this study were selected on the basis of the meridian principle described in traditional Chinese medicine [18]. Electrical stimulation was used at ST 5, 6 and 7 bilaterally (at session 2 and thereafter) and ST 3 and 4 bilaterally (at session 5 and thereafter). Si seng cong treatment point was added at session 5 and thereafter. DU 20 was chosen due to his insomnia. The needles used were sterile acupuncture needles for single use (Vinco, diameter $0.25 \mathrm{~mm}$, length 15 and $25 \mathrm{~mm}$ ). The electrical stimulator used for the electro-acupuncture was an E-stim II acupuncture stimulator unit. The frequency was placed at $30 \mathrm{~Hz}$, and the intensity was increased up to the patient's tolerated range where they can "feel the stimulus but it is not painful." The needles were left in place for 20 minutes for each session and then removed without complications. The needles were manually stimulated until a catch sensation (Deqi) and a subjective feeling of numbness, soreness and distension was felt around the selected points. The depth of needle insertion was about $0.25-1 \mathrm{~cm}$. He completed a self-report questionnaire asking about the percentage improvement of his symptoms of pain, dry mouth, difficulty swallowing, taste impairment, insomnia, and anxiety at each session.

\section{Results}

The acupuncture treatments were tolerated well with no adverse effects reported by patient. He reported resolution 
Colorado et al. Journal of Cancer Therapeutics \& Research 2013,

Table 1. Symptom improvement by acupuncture session.

\begin{tabular}{ccccc}
\hline $\begin{array}{c}\text { Acupuncture } \\
\text { Session }\end{array}$ & $\begin{array}{c}\text { Odynophagia } \\
\text { Improvement } \\
(\%)\end{array}$ & $\begin{array}{c}\text { Xerostomia } \\
\text { Improvement } \\
(\%)\end{array}$ & $\begin{array}{c}\text { Dysphagia } \\
\text { Improvement } \\
(\%)\end{array}$ & $\begin{array}{c}\text { Sense of Taste } \\
\text { Improvement } \\
(\%)\end{array}$ \\
\hline $\mathbf{1}$ & - & - & - & - \\
$\mathbf{2}$ & 50 & - & - & - \\
$\mathbf{3}$ & 95 & - & - & - \\
$\mathbf{4}$ & 95 & - & - & - \\
$\mathbf{5}$ & 100 & $10-15$ & $10-15$ & - \\
$\mathbf{6}$ & 100 & $15-20$ & $15-20$ & $15-20$ \\
$\mathbf{7}$ & 100 & $15-20$ & $15-20$ & $15-20$ \\
$\mathbf{8}$ & 100 & 25 & 25 & $15-20$ \\
$\mathbf{9}$ & 100 & 25 & 25 & 25 \\
$\mathbf{1 0}$ & 100 & $30-40$ & $25-30$ & $25-30$ \\
\hline & & & & \\
\hline
\end{tabular}

of his odynophagia after 4 acupuncture sessions, with the most dramatic improvement at session \#2 (50\%) and session \#3 (90\%). He reported gradual improvement of his overall xerostomia (in both severity and frequency), with $30-40 \%$ improvement, over 10 acupuncture sessions (Table 1). He reported feeling of thinner saliva after session $\# 5$, and reported feeling of increased amount of saliva after session \#7. He reported $20 \%$ improvement in the feeling of dry mouth during eating over 10 acupuncture sessions, but no change in the frequency of sips of liquids to aid in the swallowing of dry foods. Gradual improvement over 10 acupuncture sessions was also noted in symptoms associated with xerostomia, such as ability to swallow (25$30 \%$ improvement), difficulty speaking, and sense of taste (25-30\% improvement). He reported mild improvement in the ability to taste sweet food. He reported starting to feel a deeper sleep after session \#5, but no change in the number of hours slept at night (average of 5-6 hours). There was no change in his level of anxiety due to his dry mouth over 10 acupuncture sessions.

Four months after the 10th acupuncture session, the odynophagia has not returned. The effect of saliva increase has continued since the 10th acupuncture session, with intermittent acupuncture treatment every 4-8 weeks at the time of preparing this case study to maintain the effect.

\section{Discussion}

The combination of manual acupuncture, auricular acupuncture, and electro-acupuncture provided resolution of the patient's odynophagia, and provided significant relief of the patient's xerostomia and dysphagia. This allowed improvement in his ability to function at work, his ability to sleep, and his overall quality of life. He reported no adverse effects to acupuncture. He did not use any other medications or modalities for his symptoms of odynophagia, xerostomia, and dysphagia during his acupuncture treatments.

The results of this case study support previous studies that found acupuncture to be effective in reducing dysphagia symptom severity in head and neck cancer patients [8-11]. The results of this case study also support previous studies that found acupuncture to be effective in stimulating saliva and improving xerostomia [12-16]. This case study differs from prior studies by reporting the use of a combination of manual acupuncture, auricular acupuncture, and electro-acupuncture looking specifically at the impact on odynophagia.

The most efficient choices of points for acupuncture treatment of radiation-induced odynophagia and radiationinduced xerostomia have not yet been standardized. Prior studies have used a wide variety of acupuncture points and further study is needed to determine the efficacy of individual acupuncture points. Further study is also needed to determine if the combination of manual acupuncture, auricular acupuncture, and eletro-acupuncture utilized in this case study is more effective in treating odynophagia and xerostomia than manual acupuncture alone. This study used acupuncture points selected on the basis of the meridian principle described in traditional Chinese medicine [18]. According to Chinese medicine and Yin-Yang theory, disease will occur if the body is off-balance. Inserting needles into acupuncture points both proximally and distally will help to restore the balance and facilitate the internal energy flow or "Qi".

The mechanism of the combination approach used in this case study is unknown. Vascular and nerve damage, xerostomia, and fibrosis are all changes affected by chemoradiation [19]. Prior studies have provided evidence suggesting that acupuncture inhibits pro-inflammatory cytokines and growth factors [20]. Acupuncture has also been proposed to stimulate the autonomic nervous system, through selected afferent neurons, and cause increased parasympathetic nervous system activity that result in enhanced release of neuropeptides [21]. The inhibition of pro-inflammatory cytokines and growth factors, as well as the stimulation of the parasympathetic nervous system, may be the mechanism through which acupuncture reduces odynophagia, xerostomia, and dysphagia. The addition of auricular acupuncture and electro-acupuncture, in addition to manual acupuncture, may further activate the parasympathetic nervous system, providing additional reduction of odynophagia, xerostomia, and dysphagia.

The National Center for Complementary and Alternative Medicine is currently performing a pilot randomized, shamcontrolled trial of acupuncture for head and neck cancer patients with dysphagia after chemoradiation therapy. If the preliminary results from this pilot study are positive, a larger definitive study may be designed to fully evaluate the effects of acupuncture for head and neck cancer patients and further evaluate the underlying mechanisms on dysphagia [19].

\section{Conclusion}

Odynophagia and xerostomia are common complications after head and neck chemoradiation in oropharyngeal cancer, and can be difficult to manage. This case report found that acupuncture was effective in treating odynophagia and xerostomia after chemoradiation in oropharyngeal cancer. 


\section{Competing interests}

The authors declare that they have no competing interests.

Publication history

Received: 18-Dec-2012 Revised: 21-Jan-2013

Accepted: 31-Jan-2013 Published: 07-Feb-2013

\section{References}

1. National Cancer Institute. Head and Neck Cancers Fact Sheet, 2012. I Website

2. Bernier J, Domenge $C$, Ozsahin M, Matuszewska K, Lefebvre JL, Greiner RH, Giralt J, Maingon P, Rolland F, Bolla M, Cognetti F, Bourhis J, Kirkpatrick A, van Glabbeke M: Postoperative irradiation with or without concomitant chemotherapy for locally advanced head and neck cancer. N Engl J Med 2004, 350:1945-1952. | Article I PubMed

3. Murphy BA, Gilbert J: Dysphagia in head and neck cancer patients treated with radiation: assessment, sequelae, and rehabilitation. Semin Radiat Oncol 2009, 19:35-42. I Article I PubMed

4. Maingon P, Crehange G, Bonnetain F, Ligey-Bartolomeu A, Chamois J, Bruchon Y, Romanet P, Truc G: [Quality of life for patients treated for head and neck carcinoma]. Cancer Radiother 2010, 14:526-529. | Article | PubMed

5. Murphy BA: Clinical and economic consequences of mucositis induced by chemotherapy and/or radiation therapy. J Support Oncol 2007, 5:13-21. | Pdf | PubMed

6. Johnson JT, Ferretti GA, Nethery WJ, Valdez IH, Fox PC, Ng D, Muscoplat CC, Gallagher SC: Oral pilocarpine for post-irradiation xerostomia in patients with head and neck cancer. N Engl J Med 1993, 329:390-395. | Article | PubMed

7. Melchart D, Weidenhammer W, Streng A, Reitmayr S, Hoppe A, Ernst E, Linde K: Prospective investigation of adverse effects of acupuncture in 97733 patients. Arch Intern Med 2004, 164:104105. | Article | PubMed

8. Lu W, Posner MR, Wayne P, Rosenthal DS, Haddad RI: Acupuncture for dysphagia after chemoradiation therapy in head and neck cancer: a case series report. Integr Cancer Ther 2010, 9:284-290. | Article | PubMed Abstract | PubMed Full Text

9. Shen, H, Shen, CX: Acupuncture Treatment on "Tian Tu" (CV22) for Dysphagia in Advanced Esophageal Cancer: A 120 Cases Report. Zhejiang J Tradit Chin Med 1996, 31: 561.

10. Zheng, P, Ruan, J: Effect of Acupuncture Combined with Psychotherapy on Quality of Life in Patients with Nasopharyngeal Cancer in Post Radiation Therapy. Chin J Inf Tradit Chin Med 2002, 9: 63-64.

11. Zhou, H, Zhang, P: Effect of Swallowing Training Combined with Acupuncture on Dysphagia in Nasopharyngeal Carcinoma after Radiotherapy. Chin J Rehabil Theory Pract 2006, 12: 58-59.

12. Wu H, Wong K, Wang D: Relief of radiation-induced xerostomia with acupuncture treatment: a case presentation. $P M R$ 2011, 3:8587. | Article | PubMed

13. Wong RK, Jones GW, Sagar SM, Babjak AF, Whelan T: A Phase I-II study in the use of acupuncture-like transcutaneous nerve stimulation in the treatment of radiation-induced xerostomia in head-and-neck cancer patients treated with radical radiotherapy. Int J Radiat Oncol Biol Phys 2003, 57:472-480. | Article I PubMed

14. Jedel E: Acupuncture in xerostomia--a systematic review. J Oral Rehabil 2005, 32:392-396. I Article I PubMed

15. Blom M, Lundeberg T: Long-term follow-up of patients treated with acupuncture for xerostomia and the influence of additional treatment. Oral Dis 2000, 6:15-24. | Article | PubMed

16. Simcock R, Fallowfield L, Monson K, Solis-Trapala I, Parlour L, Langridge $C$, Jenkins V: ARIX: A randomised trial of acupuncture $v$ oral care sessions in patients with chronic xerostomia following treatment of head and neck cancer. Ann Oncol 2012. | Article | PubMed

17. Filshie J, Hester J: Guidelines for providing acupuncture treatment for cancer patients--a peer-reviewed sample policy document. Acupunct Med 2006, 24:172-182. | Article | PubMed

18. Xinnong, C. Chinese Acupuncture and Moxibustion. 1st ed. Beijing: Foreign Languages Press, 1987.

19. Lu W, Wayne PM, Davis RB, Buring JE, Li H, Goguen LA, Rosenthal DS, Tishler RB, Posner MR, Haddad RI: Acupuncture for dysphagia after chemoradiation in head and neck cancer: rationale and design of a randomized, sham-controlled trial. Contemp Clin Trials 2012, 33:700711. I Article I PubMed

20. Chen, J, et al., The effects of Acupuncture Treatment on Acute Cerebral Infarction and the Relation with Interleukin-6. Taishan Med Coll 2006, 27: 562-563.

21. Dawidson I, Angmar-Mansson B, Blom M, Theodorsson E, Lundeberg $\mathrm{T}$ : Sensory stimulation (acupuncture) increases the release of calcitonin gene-related peptide in the saliva of xerostomia sufferers. Neuropeptides 1999, 33:244-250. | Article | PubMed

\section{Citation:}

Colorado $\mathrm{B}$ and $\mathrm{Wu} \mathrm{H}$ : Acupuncture for the relief of odynophagia and xerostomia after chemoradiation therapy in oropharyngeal cancer: a case report. journal of Cancer Therapeutics and Research 2013, 2:8. http://dx.doi.org/10.7243/2049-7962-2-8 